Horizons philosophiques

\title{
Ellipse et accent rythmique (musique, poésie, peinture)
}

\section{Raymond Court}

Volume 2, numéro 1, automne 1991

De Buenos Aires à Québec

URI : https://id.erudit.org/iderudit/800882ar

DOI : https://doi.org/10.7202/800882ar

Aller au sommaire du numéro

\section{Éditeur(s)}

Collège Édouard-Montpetit

\section{ISSN}

1181-9227 (imprimé)

1920-2954 (numérique)

Découvrir la revue

Citer cet article

Court, R. (1991). Ellipse et accent rythmique (musique, poésie, peinture).

Horizons philosophiques, 2(1), 23-35. https://doi.org/10.7202/800882ar d'utilisation que vous pouvez consulter en ligne.

https://apropos.erudit.org/fr/usagers/politique-dutilisation/ 


\section{Ellipse et accent rythmique (musique, poésie, peinture)}

Ellipse, au sens premier (voir le Petit Robert), vient du grec elleipsis, signifie "manque» et désigne l'omission syntaxique ou stylistique d'un ou plusieurs mots (par exemple l'ellipse du verbe dans l'expression : "À chacun son tour»). En un sens second, et dérivé par métaphore, le mot désigne la figure géométrique à double foyer communément appelée ovale. Métaphore assurément bien significative (comme par opposition hyperbole pour excès) en tant que l'ovale témoignerait d'un manque par rapport à l'idéal de perfection représenté par le cercle avec son centre unique. D'où ce paradoxe qui fait figure de véritable renversement dialectique, celui d'un manque apparaissant comme conséquence d'un excès : posséder deux centres au lieu d'un. Sur ce point l'idéal classique hérite du MoyenÂge et de sa symbolique. II en résultera pour longtemps, on le sait, la dévalorisation du style baroque avec son goût pour l'ellipse (en architecture par exemple l'emploi de coupoles ovales comme à Saint-Charles-aux-quatre-fontaines).

L'objet de cet article sera de revenir au sens originaire du mot ellipse comme art du raccourci afin de surprendre le secret de ce pouvoir producteur de sens qu'on retrouve aussi bien sous la forme de ce qu'en poésie on nomme blanc, en peinture vide ou en musique silence. L'hypothèse ici proposée est que ce principe dynamique propre 
à l'ellipse n'est autre que l'accent rythmique lui-même à condition d'en bien pénétrer la nature exacte. Et en tout premier lieu, il importe, comme un préalable absolu pour bien apercevoir ce lien fonctionnel entre ellipse et rythme qui se trouve au fondement des correspondances profondes entre musique, poésie et peinture (mais aussi danse et architecture), de cesser de confondre le rythme et la mesure.

On trouve dans le Compendium musicae de Descartes (qui date de 1650) un exposé systématique de ce que nous nommerons la conception mensuraliste du rythme. Rien d'étonnant à cela. Le début du XVIIe siècle (ou tout au plus la fin du XVIe) a vu l'apparition de la barre de mesure qui, rappelons-le, marque la division égale de la durée par division de la ronde prise comme unité de base (la fraction placée en tête de la portée indiquant par son dénominateur, en combien de parties on a divisé la ronde et le numérateur, combien on a pris de ces parties pour constituer la mesure). Le corollaire important pour nous réside dans l'affirmation que l'accent rythmique coïncide alors avec le «temps fort", défini par son intensité et placé au premier temps de la mesure. L'analyse hégélienne du conflit du rythme et du mètre ne dira pas autre chose et, reprise par la plupart des théoriciens du $\mathrm{XIX}^{\ominus}$ siècle, elle constituera la substance même de nos solfèges «modernes» sur ce sujet 1 .

1. Ce n'est certes pas un hasard si cette thèse mensuraliste (qui renoue avec la tradition pythagoricienne) apparaît au XV|| siècle. II y a d'abord, bien sûr, la volonté clairement affirmée de définir physico-mathématiquement le son en tant que "musical» : ainsi la hauteur et les intervalles harmoniques à partir de l'expérience du monocorde selon l'axiome énoncé par Descartes, selon lequel «le son est au son comme la corde est à la corde" (l'invention postérieure de la notion de fréquence par Sauveur venant confirmer les vues cartésiennes). Musicalement, d'autre part, les complexes architectures rythmiques des polyphonistes franco-flamands ont pu conduire pour plus de commodité dans l'écriture et dans l'interprétation à inventer la barre de mesure à la manière des bornes sur la route. Mais, plus profondément, il faut, je crois, remonter jusqu'au IVe siècle après J.-C., époque à laquelle a cessé définitivement - sous la pression des langues germaniques à accent fort - d'être perçue pour la langue latine 
Plusieurs raisons ont conduit aujourd'hui à remettre en question de manière radicale cette position : à la fois les recherches sur la rythmique antique (notamment celles d'un Maurice Emmanuel) ou grégorienne (à Solesmes en tout premier lieu) et, plus largement encore, au cours de la crise qui, à l'aube du XX $X^{\ominus}$ siècle, a conduit la musique occidentale à réexaminer ses propres fondements, l'ouverture de celle-ci aux musiques orientales, extrême-orientales et africaines dont la richesse rythmique infinie échappe par sa complexité au carcan de la mesure ${ }^{2}$. Ainsi Dom André Mocquereau, aux travaux duquel renvoie Olivier Messiaen pour comprendre la nature réelle du rythme, écrit : “Nos méthodes modernes de solfège ne vont pas plus loin que l'étude de la mesure et ne peuvent s'élever jusqu'à la notion antique de mouvement rythmique qui informe toute musique, toute parole... ${ }^{3}$." Et plus loin : «ll n'existe qu'une seule rythmique générale dont les lois fondamentales, établies sur la nature humaine, se retrouvent nécessairement dans toutes les créations artistiques, musicales ou littéraires, de tous les peuples, dans tous les temps ${ }^{4}$."

Platon, dans Les lois (665 a), définissait le rythme comme l'ordonnance du mouvement et l'on sait que les Grecs qui faisaient un usage simultané des trois arts du

comme pour la langue grecque - la rythmique antique reposant sur la pesée délicate des longues et des brèves. De cette époque date, de manière irréversible, un bouleversement radical des rapports poésie/musique et désormais, la tragédie grecque, en raison de l'homologie qu'elle a connue entre la double rythmique musicale et linguistique, fera figure d'âge d'or pour le difficile régime de cohabitation qui va lui succéder.

2. II importe de noter qu'Ernest Ansermet a eu parfaitement raison de faire la distinction entre la théorie effectivement asservie au mensuralisme et le sens rythmique réellement mis en œuvre dans la musique occidentale qui y échappe en fait dans le cas d'une ujuste" interprétation (aussi bien par exemple chez Bach que chez Mozart).

3. Dom André Mocquereau, Le Nombre Musical ou Rythmique grégorienne, 2 tomes, Abbaye de Solesmes, 1927, t. I, p. 19.

4. Ibid., p. 26 
mouvement, appliquaient à la poésie et à la musique la terminologie empruntée à la danse, où l'on saisit à la source l'essence du rythme, à savoir le couple élan/repos. C'est ce que nous rapporte en toute simplicité et rigueur Dom Mocquereau :

\begin{abstract}
Dans la danse, ils appelaient elevatio (arsis) le mouvement ascensionnel, l'élan du corps, et positio, de positio (thésis) la déposition, le repos du corps au point terminus de son mouvement. En conséquence, ils appelaient arsis, élévation, élan, les sons et les syllabes qui concordaient avec l'élan des corps; et thésis, déposition, repos, les sons ou les syllabes qui se chantaient au moment même où les danseurs touchaient le sol d'où l'emploi du terme touchement pour désigner l'accent, soit pour prendre un simple appui et s'élever de nouveau, soit pour achever leur marche par un repos définitif. C'est du mouvement des danseurs que nous sont venus les termes d'arsis et de thésis. Lorsque la poésie et la musique se produisaient sans la danse, les termes d'arsis et de thésis n'étaient nullement modifiés; mais là même, ils correspondaient encore à des mouvements corporels d'élévation et d'abaissement, faits par le coryphée, le maître de chœur qui, avec le pied ou la main, indiquait les ondulations rythmiques ${ }^{5}$.
\end{abstract}

Le geste articulé sur cette dualité complémentaire de l'appui et de l'élan constitue la battue (plausus) originaire qui se noue très exactement au niveau de l'accent (ictus). Ce dernier (et en dépit de l'étymologie latine relative à l'accent déjà tonique) ne doit pas être confondu avec l'accent intensif (le "temps fort" des solfèges auquel il a été fait allusion plus haut), mais il est l'instant pulsatile qui administre le souffle (inviscéré dans la pulsation cardiaque, binaire ou ternaire selon le mode de rapport vécu au monde), à la fois en distinguant et en liant dans une unité gestuelle globale les deux mouvements contraires précédents. Le Timbalier de Paul Klee (1940, Berne) nous livre de façon saisissante en son extrême pureté hiéroglyphique 
cet instant même qui donne l'impulsion originelle au mouvement et à partir duquel, plus profondément encore, la temporalité jaillit de la subjectivité originaire (selon la leçon conjointe de saint Augustin, Kant, Husserl et Heidegger) : une rupture qui est ouverture, une coupure qui est éclatement, une césure qui est jaillissement. Ainsi le propre de cet instant remarquable est-il de nouer l'un à l'autre le présent du bondissement en avant et le présent de l'adossement au passé déjà accumulé, de façon à réaliser, en un geste indivisible mais articulé, l'unité dans le présent vivant de l'attention, des deux mouvements intentionnels opposés de l'esprit tendu (distentio ipsius animi, une distension de l'esprit lui-même, dit saint Augustin) en direction, d'une part, de l'avenir dans le présent de l'attente et, d'autre part du passé dans le présent du souvenir qui est remémoration. Ce que l'auteur des Confessions (Livre XI), devançant l'analyse husserlienne des protensions qui basculent en rétentions, a clairement analysé sur l'exemple significatif de l'exécution d'un chant, chaque séquence particulière de ce dernier étant unifiée à partir d'un rythme générateur, puis emboîté avec d'autres séquences élémentaires pour constituer un rythme plus large jusqu'à embrasser l'ensemble de la pièce ${ }^{6}$.

Le principe rythmique permet donc de rendre compte de la temporalité vécue qui est toujours structurée, c'està-dire indivisément continue (puisque l'appui et l'élan sont inséparables) et discontinue (en tant que scandée par

6. Aussi bien, tandis que les solfèges traditionnels partent d'une unité divisible et fractionnable en valeurs diverses (il s'agit de la ronde comme nous l'avons vu plus haut), la rythmique antique (et grégorienne) part d'une unité minima, indivisible mais multipliable qui est le temps premier. Celui-ci est indécomposable précisément en tant qu'il est sous-tendu par l'unité d'un acte lui-même indivisible qui est la cadence fondamentale appui/élan. Le rythme se construit alors synthétiquement à partir du temps premier par emboitement de cadences élémentaires pour atteindre à ce “rythme composé» (dessiné par le chironome) avec son élan et sa retombée à l'intérieur d'une grande phrase. Ainsi l'analyse technique du rythme que saint Augustin nous donne dans le De Musica et l'analyse du temps dans les Confessions sont rigoureusement complémentaires. 
l'alternance de l'élan et du repos). Et, si tel est déjà le temps vécu de la gestuelle quotidienne, le temps musical, en raison des propriétés de son matériau conjuguant de manière tout à fait exceptionnelle abstraction et densité matérielle, nous en offre à la fois le modèle idéal dans sa pureté extrême la plus rigoureuse et son image la plus concrète parce que la plus charnelle. La formule d'Aristoxène selon laquelle «la voix se meut lorsqu'elle chante, comme le corps lorsqu'il marche ou qu'il danse" est donc à prendre à la lettre. Ce que prouve l'importance d'une pratique comme la chironomie, sans doute aussi vieille que l'humanité, véritable "danse des mains" dont le propre est de mimer dans le détail les élans et les thésis rythmiques de chaque membre de la mélodie. Et si l'on ajoute que la chironomie, qui a tenu lieu pendant longtemps de notation musicale, a donné naissance plus tard aux neumes (de neûma: signe de la tête ou de la main), on notera avec intérêt que les recherches paléographiques d'un Dom Cardine sur les manuscrits de Laon et de SaintGall (qui remontent au $X^{\ominus}$ siècle et sont les plus achevés) montrent que le lâcher de plume dans la graphie d'un neume a une signification rythmique très précise. En clair quand la plume se lève, la dernière note est longue, c'està-dire que le levé de la plume désigne un levé rythmique, et l'on parle alors significativement de "coupure neumatique».

Cette dernière remarque nous ramène tout droit à notre problématique centrale de l'ellipse entendue comme nœud rythmique, mais pour la vérifier sur des cas concrets et en même temps l'élargir au-delà du domaine proprement musical. Car, si pour cerner la nature du rythme, nous avons fait référence surtout à la musique, c'est parce que celle-ci, en raison de son matériau de base, est sans conteste le sanctuaire du rythme, l'art où il atteint sa plus extrême rigueur et complexité. Mais il est non moins certain que le rythme est au principe de toutes les formes esthétiques et partant de ces correspondances vives entre les 
arts auxquelles il a été fait plus haut allusion. Nous développerons cette thématique du rapport ellipse/accent rythmique en l'illustrant sur trois exemples qui nous paraissent, chacun à leur manière, d'une évidence particulièrement prégnante : la peinture chinoise, la poésie mallarméenne, la musique debussyste.

La référence à la peinture chinoise offre sans doute en peinture le modèle idéal, comme en musique le chant grégorien, pour dégager dans toute sa pureté essentielle le principe rythmique lui-même, à savoir cette énergie qui anime tout l'espace pictural, comme le montrent si bien les remarques suivantes de Georges Duthuit :

Le paysagiste Song ou Yuan, au lieu de s'attacher à la définition des corps eux-mêmes, entreprend surtout de rendre l'air qui les entoure. II suffit de l'épingle d'une barque piquée sur les eaux paisibles, de la pointe d'un pic entouré de lueurs indécises, de l'agrafe entrouverte d'un bec d'hirondelle attendant la becquée, ou encore d'une griffe de fougères; c'est assez d'une traînée légère d'encre, de quelques valeurs vert malachite ou bleu lazuli : l'immensité du vide ou plutôt de l'élément fluide, pourvue d'une qualité sensible, se trouve soudain mise, d'une tache rapide ou d'un trait sûr, à la portée de notre esprit. Le trait ne résume donc pas mais suggère : pouvoir de suggestion qu'il doit à sa valeur colorante, d'une même énergie. Énergie qui peut à première vue sembler paradoxale puisqu'elle coule et, à l'instar de la couleur, n'a pas à mordre ou à griffer d'abstraites inscriptions pour féconder le chant ingrat de la feuille et de partout l'émouvoir?.

Quant à cette expérience originaire vécue à propos de l'art du pinceau, elle est d'ordre spirituel en tant qu'elle se trouve rapportée immédiatement à une vision du monde fondée dans son dynamisme profond sur la catégorie majeure de rythme. Selon la sagesse chinoise immémoriale, au commencement se trouve le Vide identifié au Souffle fondamental, principe de toute animation et, comme tel,

7. Georges Duthuit, Les Fauves, Les Trois Collines, 1949, p. 34-36. 
élément dynamique d'où jaillit l'élan rythmique (l'ictus des grégorianisants), silence générateur. C'est le Vide qui fait fonctionner le Plein en distendant les deux pôles cosmicisants, le Yin (femelle) et le Yang (mâle), auxquels correspondent respectivement la Terre et le Ciel, l'Eau et la Montagne. D'où l'art de peindre voué avant tout à revivre de l'intérieur la genèse du paysage à fin de communion avec lui et de célébration du monde. Nulle recherche donc ici de la perspective "scientifique», ni usage de la pâte, mais une restitution charnelle du "proche et du lointain" grâce au geste premier du pinceau qui en posant son trait répète le grand jeu du Vide et du Plein et inaugure à l'instant même un espace indivisément calligraphique et pictural.

Ainsi le Vide c'est le Souffle et le Souffle c'est le rythme. Et comme l'écrivait le peintre Fan Chi (fin XVIIIe siècle) :

II faut en quelque sorte que le Vide soit plus pleinement habité que le Plein. Car c'est lui qui, sous forme de fumée, de brumes, de nuages ou de souffles invisibles, porte toutes choses, les entraînant dans le processus de secrètes mutations. Loin de diluer l'espace, il confère au tableau cette unité où toutes choses respirent comme dans une structure organique ${ }^{8}$.

C'est pourquoi Georges Braque déclarait chercher à peindre "ce qui est entre la pomme et l'assiette», "le sujet» de la peinture étant «justement le rapport de ces objets entre eux et de l'objet avec l'entre-deux, c'est-à-dire les intervalles chargés d'énergie qui animent les figures ellesmêmes" ${ }^{9}$ " et atteignent à "cet état de condensation qui fait le tableau» selon Matisse. Car le Vide en question ici n'est point néant mais pulsation, donc l'élément moteur initial du rythme qu'on retrouve au principe de toute transmutation esthétique, de toute énergie créatrice de forme, que celle-ci soit picturale, poétique ou musicale.

8. Cité par Ryckmans, "Poésie et peinture», Revue d'esthétique : Autour de la Chine, $n^{\circ} 5$, Toulouse, Privat.

9. Georges Charbonnier, Le monologue du peintre, Durier, 1980, p. 20. 
Et précisément en ce qui concerne une telle correspondance des arts en relation avec le principe rythmique, est particulièrement éclairant l'exemple chinois tout à fait remarquable de l'échange permanent établi dans cette culture entre peinture et poésie grâce à la médiation de la calligraphie promue au rang d'art pilote en raison de l'écriture idéographique. D'où, d'un côté, une peinture qui, $œ u v r a n t$ avec le papier, l'encre et le pinceau, est à sa manière une écriture et nous invite à un cheminement spirituel analogue à celui que déroule le poème. Et, de l'autre, une poésie en laquelle se conjuguent attache calligraphique et fluidité syntaxique de la langue chinoise, et qui, dès lors, se présente à nous plutôt comme un aménagement spatial, nous offrant finalement un poème à voir plutôt qu'à entendre. Mais, à coup sûr, ce qui demeure dominant ici plus que jamais, c'est le principe rythmique. L'activité poétique du Vide s'exerce en effet au niveau syntaxique pour engendrer un sens métaphorique toujours plus riche et plus subtil. En supprimant des mots, en rendant la syntaxe boiteuse (au regard du langage banalisé), en montant un parallélisme entre deux sentences dont les mots se correspondent terme à terme et entrent en réciprocité par-dessus le Vide intersticiel, aussitôt la polysémie embrase le langage tout entier qui alors entre en fête et se gonfle d'un sens qui permet à l'homme de renouer avec la nature et ses rythmes profonds. Et c'est un autre chinois, Han Yu, qui compare le Souffle qui poétise ainsi les mots à la marée montante dont le flot soulève les barques échouées et les fait danser dans la lumière :

Le Souffle est comme l'eau, et les mots sont comme les objets qui flottent à la surface. Quand la quantité d'eau est suffisante, les objets grands et petits, peuvent librement se mouvoir : telle est la relation entre le souffle et les mots. Quand le souffle est

- à sa plénitude, tant la longueur des phrases que leur volume sonore atteignent leur mesure parfaite.

Or, ne retrouvons-nous pas chez Mallarmé quelque chose d'étonnamment proche aussi bien dans l'usage poé- 
tique qu'il fait de la syntaxe que dans l'intérêt croissant qu'il a porté à la typographie et qui devait déboucher sur Un Coup de dés ? Sans doute estimera-t-on que le poète outrepasse ici des possibilités ouvertes au contraire au chinois en raison de son écriture. II n'en reste pas moins que pour Mallarmé comme pour les Chinois ("c'est de l'art chinois ou japonais» s'esclaffaient les détracteurs du poète, ignorant si bien dire) le secret de la poésie consiste à introduire le Vide, d'une manière ou d'une autre, dans le trop-plein du langage de la banalité quotidienne pour l'enrichir par condensation et le faire flamber en «métaphore vive» (Ricœur). Claudel, dans une lettre à Mallarmé, sur ce point va droit à l'essentiel :

Votre phrase où, dans l'aérien contrepoids des ablatifs absolus et des incidentes, la proposition principale n'existe plus que du fait de son absence, se maintient dans une sorte d'équilibre instable et me rappelle ces dessins japonais où la figure n'est dessinée que par son blanc, et n'est que le geste résumé qu'elle trace ${ }^{10}$.

Et Claudel, toujours cité par Henri Mondor, met l'accent sur la syntaxe mallarméenne :

Il est probable pour moi que le premier élément de votre phrase en est la syntaxe ou le dessin qui des mots divers qu'elle rapproche ou distancie, de manière à les dépouiller d'une part inutile de leur sens ou à les rehausser d'un éclat étranger, constitue ce que vous appelez excellemment un terme ${ }^{11}$.

C'est ce que confirment les analyses savantes de Jacques Schérer quand il signale par exemple comme une des constantes du langage mallarméen l'ellipse du verbe (parce que trop souvent entraîné par sa fonction dans la

10. Cité par Henri Mondor dans Vie de Mallarmé, Paris, Gallimard, 1941, p. 745 .

11. Ibid., p. 710. 
mobilité du devenir), et, en conséquence, la prééminence du nom ou la substantialisation de l'adjectif, ce qui permet à cette poésie d'atteindre à une magie sonore que nous retrouverons dans la musique de Debussy et atteinte au moyen de pratiques homologues. Schérer note également le rôle capital de la ponctuation qui marque les silences et surtout, de manière générale, l'emploi toujours plus fréquent à mesure qu'on avance dans l'œuvre, de l'ellipse qui en créant des vides suscite tout un réseau d'analogies précieuses et rares.

Va également dans le même sens le souci mallarméen de la typographie. Celui-ci intervient bien antérieurement au Coup de dés et notamment dans Hérodiade et dans l'Après-midi d'un faune, et illustre parfaitement le rapport ellipse/rythme. À propos de l'emploi des blancs, Mallarmé est tout à fait explicite : "Cet artifice de typographie [offre] le repos vocal qui mesure l'élan ${ }^{12}$." Ce qui rejoint très exactement ce que nous avons dit plus haut du rythme. Et Mallarmé ajoute que les blancs ainsi insérés dans le texte du poème sont les "preuves nuptiales de l'Idée ${ }^{13}$." La structure poétique, par l'action du vide se montre alors comme déployée et ordonnée, tracée dans l'espace visible de la page :

L'armature intellectuelle du poème se dissimule et tient - a lieu - dans l'espace qui isole les strophes et parmi le blanc du papier : significatif silence qu'il n'est pas moins beau de composer que des vers ${ }^{14}$.

Cette volonté de visualisation du poétique par inscription des rythmes dans l'espace se traduit chez Mallarmé par une double hantise : celle de la partition musicale et

12. Stéphane Mallarmé, CEuvres complètes, Paris, Gallimard, «Pléiade», 1945, p. 407.

13. Ibid., p. 387.

14. Ibid., p. 872. 
celle du mime. D'un côté, il s'agit, en séparant par des blancs des groupes de mots ou des mots entre eux, de scander le mouvement poétique selon la "vision spontanée de la page» ou selon la «ligne parfaite» du Vers ${ }^{15}$. De l'autre, grâce à cette "forme théâtrale de poésie par excellence ${ }^{16}$ ", il s'agit de créer un espace scénique comme le vide à distance sur lequel, grâce aux signes tracés "en blanc et noir" (on pense au titre d'une des ultimes pièces pour piano de Debussy) par les danseurs, «fleurira la figu$\mathrm{re}^{17}$ " «instituant un lieu 18 " propice au Songe : «le Mime .. installe . . un milieu, pur, de fiction ${ }^{19}$."

Debussy, déjà plusieurs fois évoqué nous ramène à la musique avec laquelle nous avions inauguré notre réflexion axée sur le rythme. Et comment mieux conclure qu'avec ce musicien dont on sait les affinités déclarées avec le poète du Faune? Nul d'ailleurs autant que lui n'a en ce début de siècle révolutionné son art jusqu'aux fondements. En l'absence d'une structure centrée de l'œuvre par suite de l'éclatement tonal (obtenu lui-même par subversion modale ou harmonique), cette musique, totalement non-discursive, a réussi à libérer la forme de toute thématique obligatoire (au point qu'on a pu parler à son propos d'anti-développement) pour cultiver les «sonorités opposées" (c'est le titre même d'une des Études) dans leur matérialité pure, en cherchant en quelque sorte le son sous la note. Aussi se présente-t-elle à nous éclatée, elliptique, pleine de silences (comme la peinture chinoise est pleine de vide), réalisant le paradoxe suprême d'une multiplicité pure et pourtant sans éparpillement. Ainsi, dans Jeux, partition suprême à cet égard, à travers des transformations

15. Ibid., p. 455.

16. Ibid., p. 308.

17. Ibid., p. 308.

18. Ibid., p. 309.

19. Ibid., p. 310. 
incessantes avec des poussées dynamiques puis des retombées, de brusques éclairages suivis d'assombrissements non moins soudains, mystérieusement l'unité organique de l'œuvre demeure grâce à une liberté rythmique qui retrouve la souplesse érotico-mystique si sensuelle du gamelan balinais avec ses percussions douces.

En tout art finalement ce que Flaubert nomme «la forme interne du style" réside en dernière analyse dans l'énergie du non-dit, au cœur de ce vide central où réside la vérité inapprochable et indicible, ce dont témoigne par symbole le thème, inlassablement répété par les peintres et poètes chinois, de l'ermitage désert mais où précisément l'absence même du Maître nous ouvre, par illumination soudaine du paysage environnant, la voie vers le comble de la présence :

Sous le sapin, j'interroge le disciple :

"Le maître est parti chercher des simples,

Par-là, au fond de cette montagne.

Nuages épais : on ne sait plus où...»

Chia Tao $\left(\mathrm{IX}^{\mathrm{e}} \text { siècle }\right)^{20}$

Ou encore ce mot d'un Chinois : «Tout ce qui peut s'énoncer est dénué d'importance». Ce qui doit nous rendre bien modestes!

Raymond Court

Université Jean Moulin — Lyon III

20. Cité par François Cheng dans L'espace du rêve. Mille ans de peinture chinoise, Paris, Phébus, 1980, p. 108. 